\title{
Upregulation of glutamate metabolism by BYHWD in cultured astrocytes following oxygen-glucose deprivation/reoxygenation in part depends on the activation of p38 MAPK
}

\author{
PENG YU $^{1}$, LI GUAN ${ }^{1}$, LEQUAN ZHOU ${ }^{1}$, JIANCHAO GUO ${ }^{1}$, RUIXIAN GUO ${ }^{2}$, \\ RUISHAN LIN ${ }^{1}$, WENTING DING ${ }^{1}$, XIAOYING LI ${ }^{1}$ and WEI LIU ${ }^{1}$ \\ ${ }^{1}$ Department of Physiology, College of Fundamental Medical Science, Guangzhou University of Chinese Medicine, \\ Guangzhou, Guangdong 510006; ${ }^{2}$ Department of Physiology, Zhongshan Medical College, \\ Sun Yat-sen University, Guangzhou, Guangdong 510080, P.R. China
}

Received January 28, 2016; Accepted January 26, 2017

DOI: $10.3892 /$ etm.2017.4330

\begin{abstract}
Recent studies have demonstrated that Buyang Huanwu Decoction (BYHWD) decreased glutamate levels subsequent to cerebral ischemia. Glutamate transporter-1 (GLT-1) and glutamine synthetase (GS), which are located in astrocytes, mainly contribute to glutamate transportation, thus reducing glutamate concentration. BYHWD has previously been demonstrated to upregulate GLT-1 and GS following ischemia in vivo. However, whether BYHWD can directly influence astrocytic GLT-1/GS levels remains unknown. In the present study, the effect of BYHWD containing serum (BYHWD-CS) on GLT-1/GS levels in astrocytes following oxygen-glucose deprivation/reoxygenation (OGD/R) was investigated. The results revealed that BYHWD-CS enhanced the expression levels of GLT-1 and GS in cultured astrocytes, which reduced glutamate concentration in the culture medium. Meanwhile, increased p38 mitogen-activated protein kinase (p38 MAPK) was phosphorylated (activation form) by BYHWD-CS in cultured astrocytes, and the specific p38 inhibitor SB203580 blocked the increase of GLT-1/GS accompanied by decreased
\end{abstract}

Correspondence to: Dr Wei Liu, Department of Physiology, College of Fundamental Medical Science, Guangzhou University of Chinese Medicine, 232 Waihuan East Road, Panyu, Guangzhou, Guangdong 510006, P.R. China

E-mail: weiliu1980@yahoo.com

Abbreviations: BYHWD, buyang huanwu decoction; GLT-1, glutamate transporter-1; GS, glutamine synthetase; CS, containing serum; OGD/R, oxygen-glucose deprivation/reoxygenation; GFAP, glial fibrillary acidic protein; MAPKs, mitogen-activated protein kinases; ERK, extracellular signal-regulated kinase; JNK, c-Jun amino-terminal kinase; ROS, reactive oxygen stress

Key words: Buyang Huanwu Decoction, oxygen-glucose deprivation/reoxygenation, glutamate transporter-1, glutamine synthetase, p38 mitogen-activated protein kinase, glutamate, glial fibrillary acidic protein cell viability. Furthermore, SB203580 suppressed the effect of BYHWD-CS on the level of glial fibrillary acidic protein (an astrocytic marker), thus confirming that astrocytes are directly involved in the protective role of BYHWD after OGD/R. These findings suggest that BYHWD upregulates GLT-1 and GS via p38 MAPK activation, and protects cultured astrocytes from death caused by OGD/R (typical in vitro model), which complemented the role of astrocytes in the protective effect of BYHWD.

\section{Introduction}

Buyang Huanwu Decoction (BYHWD), originating from 'Yi Lin Gai Cuo', is a traditional Chinese herbal prescription that has been applied in the treatment of cerebral ischemia in China for hundreds of years (1). Systematic reviews and meta-analyses of BYHWD have demonstrated that BYHWD is generally safe and possesses substantial neuroprotective action in patients and various animal models of focal ischemia $(2,3)$. The protective effect of BYHWD against focal ischemia involves multiple mechanisms, for instance, reducing calcium overload injury, attenuating reactive oxygen stress (ROS) (4), inhibiting the inflammatory response and apoptosis (5), and promoting nerve regeneration and synapse formation (6).

Increasing evidence has demonstrated that BYHWD decreases the glutamate concentration in animal models following focal cerebral ischemia/reperfusion $(7,8)$. Glutamate is a pivotal cause for ischemia injury, as it can induce high levels of intracellular calcium, which induces mitochondrial dysfunction, ROS accumulation and nitric oxide release (9). Previous studies have demonstrated that glutamate transportation is the predominant way for clearance of extracellular glutamate and limiting excitotoxicity (10). Notably, gene deletion, gene knockdown, and pharmacological studies have indicated that the glutamate transporter-1 (GLT-1) subtype may contribute up to $90 \%$ of total transport in the forebrain, while inhibition of GLT-1 leads to severe neurological deficits and larger infarction volume (10,11). Furthermore, the intracellular glutamate is degraded by glutamine synthetase (GS), which accelerates glutamate transportation (12). Inhibition 
of GS significantly impaired glutamate uptake and resulted in increased excitotoxicity to neurons (13). Therefore, GLT-1 and GS contribute to glutamate metabolism collectively. Our previous study observed that BYHWD upregulated the expression levels of GLT-1 and GS following focal ischemia (14). Notably, these two proteins are mainly located in astrocytes $(14,15)$; therefore, astrocytes are the major cells for degrading glutamate. Whether BYHWD directly influences the astrocytic GLT-1/GS levels (through neurons or other types of cells) is not fully understood, and the potential mechanisms remain unknown.

Nine bioactive components (including astragaloside I, astragaloside II, astragaloside IV, formononetin, ononin, calycosin, calycosin-7-O- $\beta$-d-glucoside, ligustilide and paeoniflorin) have been identified in the plasma subsequent to BYHWD treatment (16). Therefore, it is difficult to use only specific components of the formula to investigate the protective role of BYHWD. However, BYHWD-containing serum (BYHWD-CS) has been developed and is generally accepted for use in traditional Chinese medicine studies (1).

One of the most important mechanisms underlying glutamate metabolism is the activation of mitogen-activated protein kinases (MAPKs), which include extracellular signal-regulated kinase (ERK), p38 MAPK and c-Jun amino-terminal kinase (JNK) (17). There are numerous studies demonstrating that these signaling molecules are activated following focal cerebral ischemia $(18,19)$.

In the present study, primary cultured astrocytes were treated with oxygen-glucose deprivation/reoxygenation (OGD/R). The current study also investigated whether BYHWD-CS directly enhanced the glutamate metabolism by affecting the GLT-1 and GS levels in cultured astrocytes following OGD/R. Furthermore, the study examined whether phosphorylation of MAPKs mediated the upregulation of astrocytic GLT-1/GS levels induced by BYHWD-CS treatment.

\section{Materials and methods}

Preparation of BYHWD-CS. BYHWD was obtained from Guangdong Provincial Traditional Chinese Medical Hospital, which is the second affiliated hospital of Guangzhou University of Chinese Medicine (Guangzhou, China). The decoction is composed of Radix Astragali (Gansu, China), Radix Angelicae sinensis (Gansu, China), Radix Paeonia Rubra (Sichuan, China), Rhizoma Chuanxiong (Sichuan, China), Semen Persicae (Hebei, China), Flos Carthami (Sichuan, China) and Lumbricus (Guangxi, China). These herbs were mixed at proportions of 120:6:4.5:3:3:3:3 (20). The components were immersed in distilled water, decocted twice (each time for $30 \mathrm{~min}$ ), and then the filtered liquid was condensed into the crude drug concentration of $2 \mathrm{~g} / \mathrm{ml}$.

Animals and treatment. Adult male Sprague-Dawley (SD) rats (weight, 220-250 g) were purchased from the Experimental Animal Center of the Guangzhou University of Chinese Medicine (Guangzhou, China). All the experimental procedures were conducted in accordance with the National Institutes of Health guidelines for the Care and Use of Laboratory Animals and approved by the Animal Care and Use Committee of the
Guangzhou University of Chinese Medicine (Guangdong, China; ds20130001). A total of 42 rats were housed individually with ad libitum access to water and food in a temperature $\left(22 \pm 2^{\circ} \mathrm{C}\right)$ and light controlled $(12 \mathrm{~h}$ light/dark cycle) quiet room at a humidity of $65-75 \%$. The rats were randomized into two groups (Control and BYHWD treatment, $\mathrm{n}=20$ for Control, $\mathrm{n}=22$ for BYHWD). Rats in one group were intragastrically administered with BYHWD $18 \mathrm{~g} / \mathrm{kg}$ twice a day for consecutive 3 days (13), while the other group was treated with distilled water as the vehicle. At $1 \mathrm{~h}$ after the last administration, blood was obtained via cardiac puncture from each group, and defined as the BYHWD-containing serum (BYHWD-CS) and vehicle containing serum (vehicle-CS) samples, respectively. All samples were heated at $56^{\circ} \mathrm{C}$ for $30 \mathrm{~min}$ to be inactivated, then filtered and kept at $-20^{\circ} \mathrm{C}$ until further use (16).

Primary culture of cortical astrocytes. Primary astrocytes were prepared from newborn (1-2 days) SD rats as described previously (21). In brief, newborn rats were sacrificed by cervical dislocation, and whole brains were extracted. Cerebral cortices were separated, minced, and trypsinized. The cell pellet was suspended in Dulbecco's modified Eagle's medium (DMEM)/F12 (Gibco; Thermo Fisher Scientific, Inc., Waltham, MA, USA) containing $10 \%$ fetal bovine serum (FBS; Hyclone; GE Healthcare Life Sciences, Logan, UT, USA) and plated in Nunc flasks. Cultures were maintained in a $37^{\circ} \mathrm{C}$ incubator with $5 \% \mathrm{CO}_{2}$, and the culture medium was replaced every 3 days. When the cells reached confluence (75-80\%), the flasks were shaken vigorously for further purification. The third passage of astrocytes was generally used for experiments. Astrocytes were assessed by glial fibrillary acid protein (GFAP; 1:800, cat. no. ab7260; Abcam, Cambridge, MA, USA) immunocytochemistry and samples with a purity of $>95 \%$ were used in subsequent experiments.

$O G D / R$ and BYHWD-CS treatment. A model of OGD/R was established in astrocytes according to the following procedure. Briefly, astrocytes were initially rinsed and incubated with an isotonic OGD/R solution (containing $0 \mathrm{mM}$ glucose, $21 \mathrm{mM}$ $\mathrm{NaHCO}_{3}, 120 \mathrm{mM} \mathrm{NaCl}, 5.36 \mathrm{mM} \mathrm{KCl}, 0.33 \mathrm{mM} \mathrm{Na}_{2} \mathrm{HPO}_{4}$, $0.44 \mathrm{mM} \mathrm{KH}_{2} \mathrm{PO}_{4}, 1.27 \mathrm{mM} \mathrm{CaCl}_{2}$ and $0.81 \mathrm{mM} \mathrm{MgSO}_{4}, \mathrm{pH}$ 7.4) in a hypoxic instrument (Billups-Rothenberg, Inc., San Diego, CA, USA) for $2 \mathrm{~h}$ in an atmosphere with $94 \% \mathrm{~N}_{2}, 1 \% \mathrm{O}_{2}$ and $5 \% \mathrm{CO}_{2}(22)$. Astrocytes were treated for 12,24 and $48 \mathrm{~h}$ in the following groups: Control group, treated with DMEM/F12 with $10 \%$ FBS only; OGD/R group, treated with OGD/R and DMEM/F12 supplemented with 10\% FBS; BYHWD-CS group, treated with OGD/R and DMEM/F12 with $10 \%$ BYHWD-CS; vehicle-CS group, treated with OGD/R and DMEM/F12 with $10 \%$ vehicle-CS. For signaling pathway investigation, in order to confirm the role of p38 MAPK in the upregulation of GLT-1/GS, a specific inhibitor of p38 MAPK (SB203580, $10 \mu \mathrm{M}$; Selleck Chemicals, Houston, TX, USA) was added into culture medium during reperfusion.

Western blot analysis. Subsequent to the OGD/R experiments, the cells were collected and lysed to extract the protein content. Next, the protein concentration was quantified with a BCA assay kit (cat. no. 23225, Pierce; Thermo Fisher Scientific, Inc., Waltham, MA, USA). 
Protein samples were fractionated by $10 \%$ sodium dodecyl sulphate-polyacrylamide gel electrophoresis and then electrotransferred to polyvinylidene difluoride membranes. The membranes were blocked for $1 \mathrm{~h}$ with $0.1 \%$ Tween-20 in Tris-buffered saline (TBS-T) containing 5\% fat-free milk and incubated overnight at $4{ }^{\circ} \mathrm{C}$ with primary antibodies. The antibodies used were as follows: Anti- $\beta$-actin (1:20,000; cat. no. A2668) obtained from Sigma-Aldrich (St. Louis, MO, USA); anti-GLT-1 (1:1,000; cat. no. ab41621), anti-GS $(1: 2,000$; cat. no. ab73593) and anti-GFAP $(1: 2,000$; cat. no. MAB360), which were purchased from Abcam; anti-p38 MAPK (1:1,000; cat. no. 8690), anti-p-p38 MAPK (1:1,000; cat. no. 4511), anti-JNK (1:1,000; cat. no. 9258), anti-p-JNK (1:1,000; cat. no. 4668), anti-ERK (1:2,000; cat. no. 4695) and anti-p-ERK (1:2,000; cat. no. 4370), purchased from Cell Signaling Technology, Inc. (Boston, MA, USA). Subsequently, the membranes were probed with horseradish peroxidase-conjugated goat anti-rabbit (or goat anti-mouse) secondary antibody (1:1,000; cat. nos. HAF008 and HAF007; R\&D Systems, Boston, MA, USA) in TBS-T with $3 \%$ fat-free milk. Finally, the membranes were developed in an enhanced chemiluminescence solution (EMD Millipore, Billerica, MA, USA) and exposed onto an X-ray film. For quantification, the gray values of each group were measured and normalized with the control band by Image $\mathrm{J}$ software (version 1.46r; National Institutes of Health, Bethesda, MD, USA).

Glutamate concentration measurement. The glutamate level in the culture media was measured with the Glutamate Assay kit (Abnova, Taipei, Taiwan) (23). Briefly, $0.2 \mathrm{ml}$ culture media was collected after the indicated treatments, and corresponding reagents were added in accordance with the manufacture's protocol. The absorbance was measured at $565 \mathrm{~nm}$ with a microplate reader (PerkinElmer, Inc., Fremont, CA, USA). Glutamate level was calculated on the basis of the standard curve in each experiment.

Cell viability assay. Cell counting kit-8 assay (Nanjing Jiancheng Bioengineering Institute, Nanjing, China) was used to estimate the cell viability of cultured astrocytes. Following different treatments, astrocytes were incubated for $4 \mathrm{~h}$ with $10 \mu \mathrm{l} \mathrm{CCK}-8$ solution. The absorbance at $450 \mathrm{~nm}$ was determined by a microplate reader. The percentage of cell viability was calculated according to the optical density (OD) as follows: Cell viability $(\%)=(\mathrm{OD}$ treatment group/OD control group) $\mathrm{x} 100 \%$.

Statistical analysis. Values are presented as the mean \pm standard error. Significant differences between groups were analyzed by one-way analysis of variance. The Student's t-test was used to compare the means of samples in the two groups. All analyses were performed with SPSS version 16.0 for Windows (SPSS, Inc., Chicago, IL, USA). Differences were considered as statistically significant when $\mathrm{P}<0.05$.

\section{Results}

BYHWD treatment enhances the expression of GLT-1 and $G S$. GLT-1 and GS expression levels were evaluated at
12, 24 and $48 \mathrm{~h}$ after OGD/R. Fig. 1A shows that treatment with $\mathrm{OGD} / \mathrm{R}$ at the aforementioned time points markedly reduced GLT-1 expression compared with the control group $(\mathrm{P}<0.05)$. However, BYHWD-CS significantly upregulated GLT-1 expression at $24 \mathrm{~h}$ after OGD/R $(\mathrm{P}<0.05)$, while vehicle-CS did not have a significant effect, indicating that BYHWD-CS treatment increased the expression of GLT-1. By contrast, there was no significant difference between the BYHWD-CS and vehicle-CS groups at 12 and $48 \mathrm{~h}$ after OGD/R, suggesting that the influence of BYHWD-CS on GLT-1 was only evident after $\sim 24 \mathrm{~h}$, but could not be sustained for $48 \mathrm{~h}$.

Similar with the GLT-1 expression findings, GS expression was decreased at 12, 24 and $48 \mathrm{~h}$ after OGD/R compared with that in the control group ( $\mathrm{P}<0.05$; Fig. 1B). However, GS expression was upregulated by BYHWD-CS at $12 \mathrm{~h}$, peaked at $24 \mathrm{~h}$ and decreased gradually thereafter. There was a significant difference between the BYHWD-CS and vehicle-CS groups only at $24 \mathrm{~h}$ after OGD/R, but not at 12 or $48 \mathrm{~h}$ (Fig. 1B). Based on these results, OGD/R at $24 \mathrm{~h}$ was selected for the subsequent experiments.

Upregulation of GLT-1 and GS by BYHWD-CS contributes to the decreased glutamate concentration. In order to determine whether the upregulated GLT-1 and GS as a result of BYHWD-CS treatment contribute to decreased glutamate concentration, the glutamate level in culture media was measured. As shown in Fig. 1C, OGD/R induced glutamate release compared with the control group $(\mathrm{P}<0.05)$. However, BYHWD-CS suppressed this effect $(\mathrm{P}<0.05)$, whereas vehicle-CS did not have a significant effect on glutamate compared with the $\mathrm{OGD} / \mathrm{R}$ model group, indicating that BYHWD-CS can indeed decrease glutamate concentration via GLT-1/GS upregulation.

p38 MAPK activation is involved in the upregulation of GLT-1 and GS after BYHWD treatment. To investigate the involvement of the MAPK family of proteins in the effect of BYHWD-CS treatment after OGD/R, the levels of three members (p-ERK, p-p38 MAPK and p-JNK) were initially detected, with the phosphorylated form of the proteins indicating activation. As shown in Fig. 2, the expression levels of p-ERK, p-p38 MAPK and $\mathrm{p}-\mathrm{JNK}$ were increased in the OGD/R group $(\mathrm{P}<0.05)$; however, no evident difference was observed in the p-ERK and p-JNK levels between the BYHWD-CS and vehicle-CS groups. Notably, BYHWD-CS treatment significantly upregulated p-p38 MAPK $(\mathrm{P}<0.05)$, while vehicle-CS did not have a significant effect, suggesting that BYHWD-CS was able to increase the expression of p-p38 MAPK.

To confirm the role of $\mathrm{p} 38$ MAPK in the upregulation of GLT-1/GS, SB203580, a specific inhibitor of p38 MAPK was used. Fig. 3 shows that GLT-1 and GS expression levels were inhibited following OGD/R $(\mathrm{P}<0.05)$. Treatment with vehicle-CS alone did not produce any effect compared with the OGD/R group; however, BYHWD-CS upregulated their expression $(\mathrm{P}<0.05)$. Coadministration of SB203580 and BYHWD-CS significantly reduced the levels of GLT-1 and GS compared with the BYHWD-CS group $(\mathrm{P}<0.05)$, indicating that SB203580 downregulated the expression of GLT-1/GS, which suggested that the p38 MAPK signaling pathway may 
A

Control OGD/R Vehicle-CS BYHWD-CS

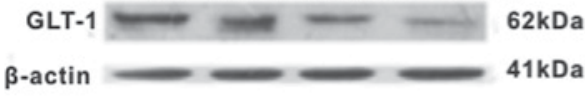

$24 \mathrm{~h}$

Control OGD/R Vehicle-CS BYHWD-cS

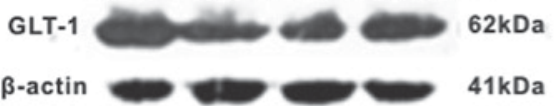

$48 \mathrm{~h}$

Control OGD/R Vehicle-CS BYHWD-CS

GLT-1 $=62 \mathrm{kDa}$

$\beta$-actin $=20=41 \mathrm{kDa}$

B
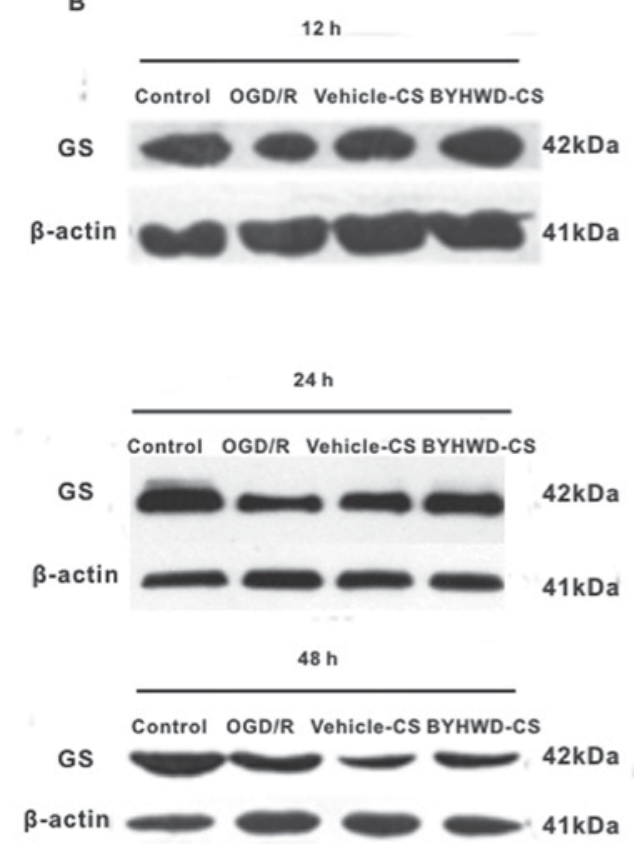

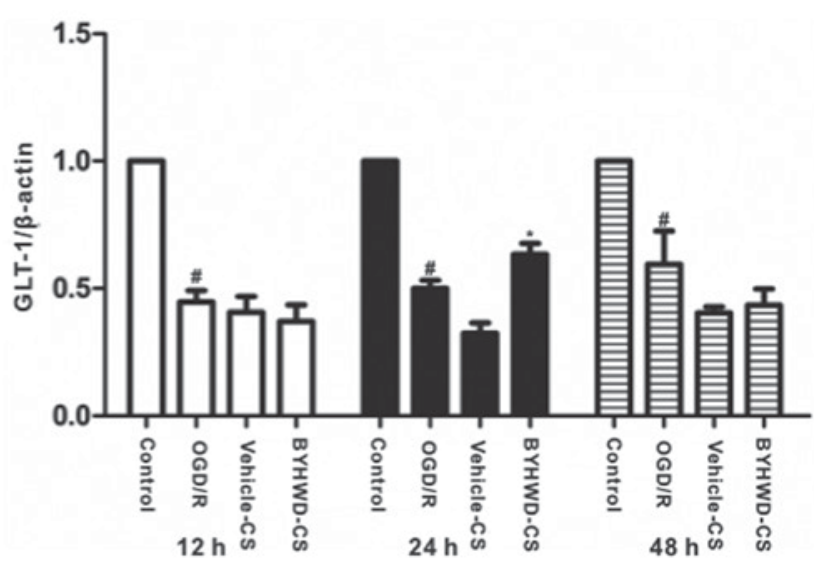

Groups

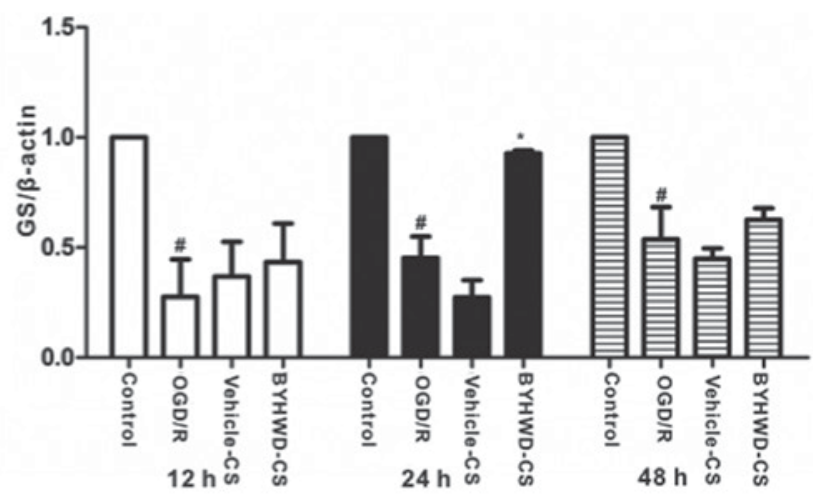

Groups

C

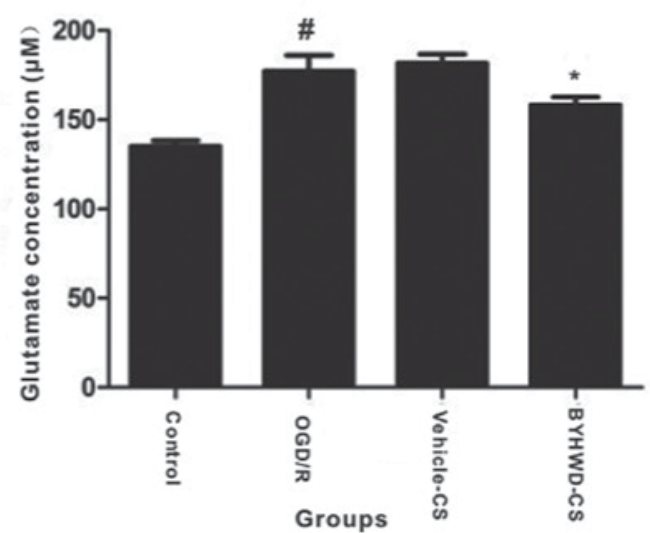

Figure 1. BYHWD-CS decreased glutamate level by increasing the expression of GLT-1 and GS in the rat cortical astrocytes. Western blots and quantified levels of (A) GLT-1 expression and (B) GS expression are shown. After OGD/R, GLT-1 and GS levels were upregulated by BYHWD-CS at 24 h compared with the vehicle-CS group. (C) Glutamate assay confirmed that the upregulated GLT-1/GS contributed to lower glutamate in the culture supernatant. $\mathrm{n}=5$. ${ }^{*} \mathrm{P}<0.05$ vs. control group; *P<0.05 vs. vehicle-CS group. BYHWD-CS, Buyang Huanwu Decoction-containing serum; GLT-1, glutamate transporter-1; GS, glutamine synthetase; OGD/R, oxygen-glucose deprivation/reoxygenation. 

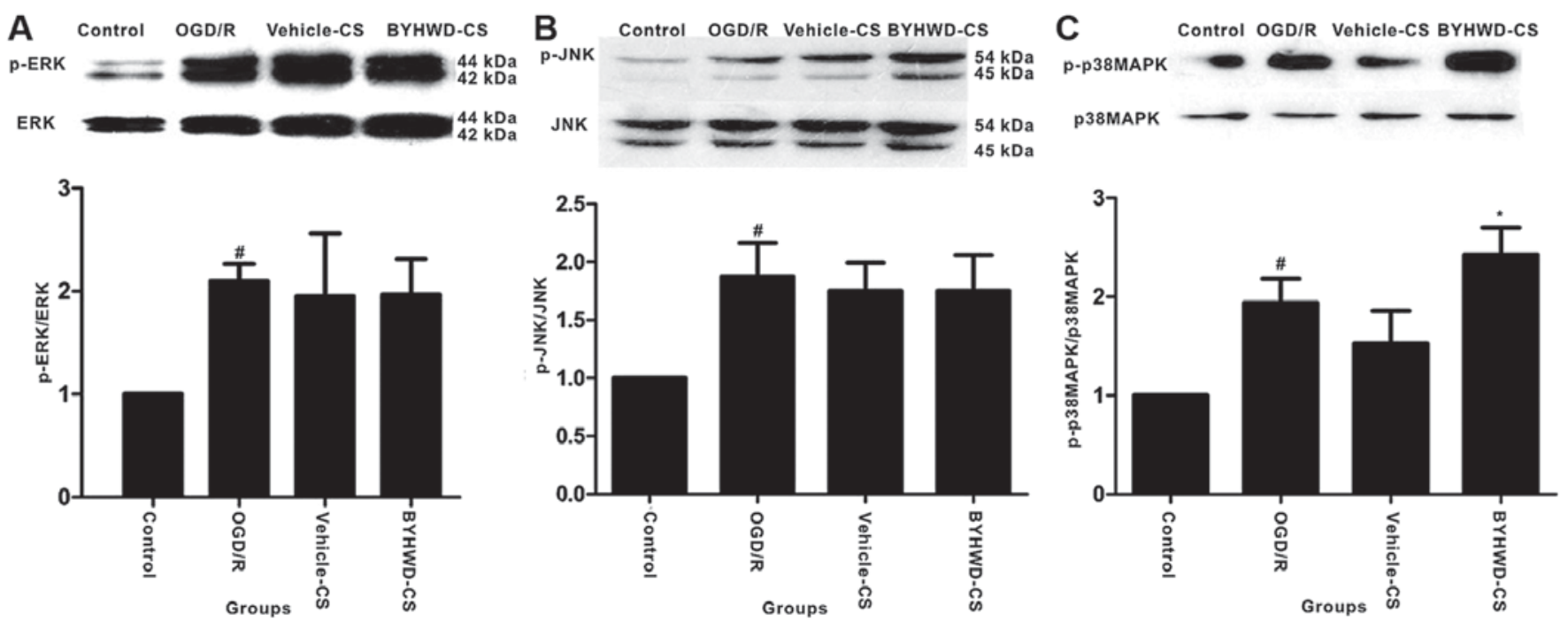

Figure 2. Effect of BYHWD-CS on the phosphorylation of the MAPKs (A) ERK, (B) JNK and (C) p38 MAPK in cultured astrocytes. BYHWD-CS did not have a significant effect on the phosphorylation of ERK or JNK in astrocytes following OGD/R, when compared with the vehicle-CS group. By contrast, BYHWD-CS markedly upregulated p-p38 MAPK in astrocytes following OGD/R compared with the vehicle-CS group. $n=4$. " $\mathrm{P}<0.05$ vs. control group; ${ }^{*} \mathrm{P}<0.05$ vs. vehicle-CS group. BYHWD-CS, Buyang Huanwu Decoction-containing serum; OGD/R, oxygen-glucose deprivation/reoxygenation; MAPKs, mitogen-activated protein kinases; ERK, extracellular signal-regulated kinase; JNK, c-Jun amino-terminal kinase.

A
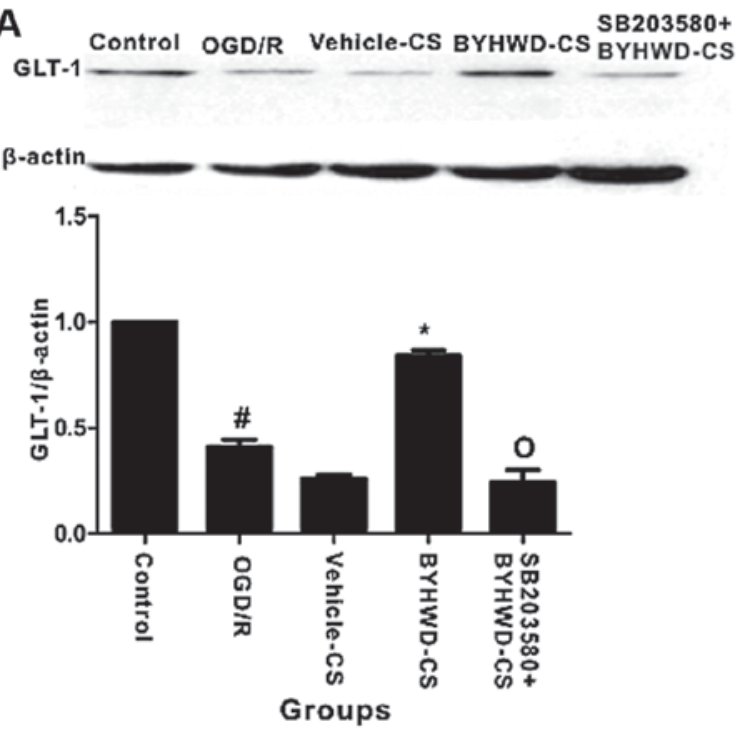

B
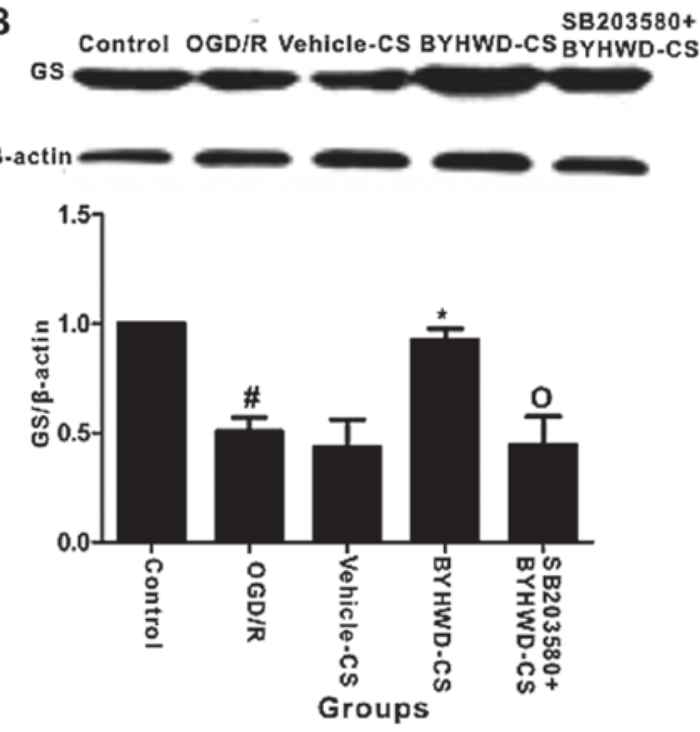

Figure 3. Effect of SB203580 (a p38 MAPK specific inhibitor) combined with BYHWD-CS on the level of (A) GLT-1 and (B) GS in cultured astrocytes following OGD/R. Coadministration of SB203580 with BYHWD-CS significantly inhibited the upregulation of GLT-1 and GS induced by BYHWD-CS. n=5. ${ }^{\#} \mathrm{P}<0.05$ vs. control group; ${ }^{*} \mathrm{P}<0.05$ vs. vehicle-CS group; ${ }^{\circ} \mathrm{P}<0.05$ vs. BYHWD-CS group. BYHWD-CS, Buyang Huanwu Decoction-containing serum; GLT-1, glutamate transporter-1; GS, glutamine synthetase; OGD/R, oxygen-glucose deprivation/reoxygenation.

serve a role in upregulating GLT-1/GS levels through the BYHWD-CS treatment following OGD/R.

Cell viability following $O G D / R$. As shown in Fig. 4, OGD/R resulted in significantly decreased cell viability compared with the control group $(\mathrm{P}<0.05)$; however, this influence of $\mathrm{OGD} / \mathrm{R}$ was significantly inhibited by BYHWD-CS treatment (Fig. 4; $\mathrm{P}<0.05$ ), suggesting the protective effect of BYHWD-CS. Furthermore, coadministration with SB203580, a p38 MAPK inhibitor, markedly blocked the effect of BYHWD-CS (Fig. 4; $\mathrm{P}<0.05$ ), while vehicle-CS alone had no significant effect compared with the OGD/R model group.

SB203580 affected astrocytic marker GFAP expression. As shown in Fig. 5, GFAP expression was upregulated by OGD/R $(\mathrm{P}<0.05)$, which was significantly attenuated subsequent to BYHWD-CS treatment $(\mathrm{P}<0.05)$. In addition, co-administration of SB203580 blocked this effect of BYHWD-CS $(\mathrm{P}<0.05)$, 


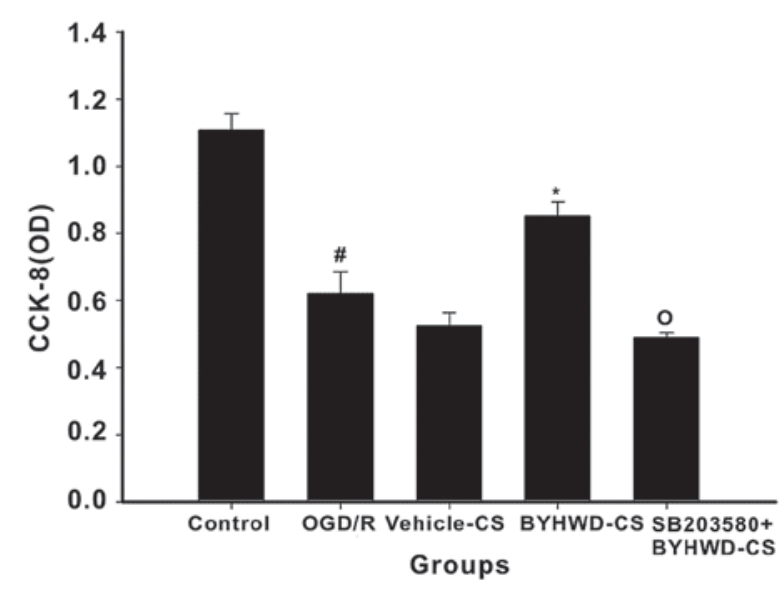

Figure 4. Effect of BYHWD-CS alone or combined with SB203580 on the viability of cultured astrocytes following $\mathrm{OGD} / \mathrm{R}$ was determined by cell counting kit- 8 assay. OGD/R evidently decreased the astrocytic viability; however, treatment with BYHWD-CS significantly attenuated the cytotoxicity induced by OGD/R. By contrast, SB203580 blocked the protective effect of BYHWD-CS in cultured astrocytes. $n=5 .{ }^{~} \mathrm{P}<0.05$ vs. control group; ${ }^{*} \mathrm{P}<0.05$ vs. vehicle-CS group; ${ }^{\circ} \mathrm{P}<0.05$ vs. BYHWD-CS group. BYHWD-CS, Buyang Huanwu Decoction-containing serum; OGD/R, oxygen-glucose deprivation/reoxygenation.

while vehicle-CS treatment alone did not have a marked effect on GFAP expression compared with the OGD/R model group.

\section{Discussion}

The present study demonstrated that BYHWD-CS contributed to the increased glutamate metabolism by upregulating GLT-1 and GS. Similarly to the current results, numerous other drugs or treatments, including beta-lactam antibiotics or ischemic preconditioning, were also reported to have a protective effect by upregulation of glutamate transportation (24-26). The present in vitro experiments further illustrated that BYHWD directly affected astrocytic GLT-1 and GS regardless of the neuron existence.

Although the p38 MAPK pathway is widely considered to be involved in cerebral ischemia, its exact role is controversial. Various studies have demonstrated that activation of the p38 MAPK signaling pathway contributed to the inflammatory response, which accelerated cellular apoptosis $(27,28)$, therefore having a harmful role in stroke. However, there are also certain experiments documenting the beneficial role of the p38 MAPK signaling pathway, such as a study reporting that pretreatment with p38 inhibitor SB203580 aggravated ischemic injury and vascular leakage (29). Furthermore, activation of p38 MAPK was reported to be associated with the protective action of isoflurane preconditioning, sevoflurane and ischemic tolerance (30-32). Further studies have demonstrated that phosphorylation of p38 MAPK exhibited a protective role against cerebral ischemic injury via mitochondria translocation of Bcl-xL (33). Thus, the p38 MAPK pathway potentially contributed not only to inflammation, but also to other signaling transduction, such as the Bcl-xL (33). The findings of the present study demonstrated that phosphorylation of p38 MAPK participated in the upregulation of GLT-1 and GS, thus leading to decreased glutamate, which

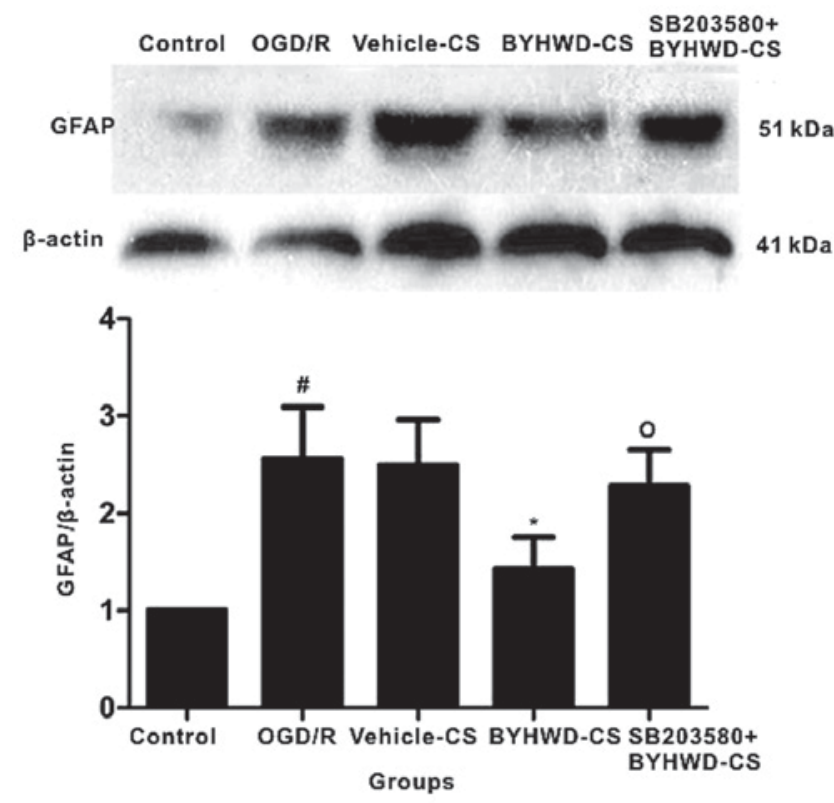

Figure 5. Effect of SB203580 on the expression of GFAP (an astrocytic marker) in the culture following $\mathrm{OGD} / \mathrm{R}$, as determined by western blot analysis. OGD/R markedly upregulated GFAP expression compared with the control group. BYHWD-CS significantly inhibited the increase of GFAP compared with the vehicle-CS group, whereas SB203580 significantly inhibited the effect of BYHWD-CS in cultured astrocytes following OGD/R. $n=5 .{ }^{*} \mathrm{P}<0.05$ vs. control group; " $\mathrm{P}<0.05$ vs. vehicle-CS group; ${ }^{\circ} \mathrm{P}<0.05$ vs. BYHWD-CS group. BYHWD-CS, Buyang Huanwu Decoction-containing serum; OGD/R, oxygen-glucose deprivation/reoxygenation; GFAP, glial fibrillary acidic protein.

provided new evidence for the protective role of p38 MAPK activation.

Another important finding in the current study was that BYHWD attenuated the cytotoxicity induced by OGD/R injury. It was speculated that one of the mechanisms may be associated with upregulated GLT-1/GS, since SB203580, which blocked upregulation of GLT-1/GS, inhibited this effect of BYHWD on astrocytic viability and activation. Notably, it is well documented that astrocytes serve critical roles in cerebral ischemia, such as in the regulation of water and ion homeostasis, cerebral blood flow, maintenance of the blood-brain barrier, control of extracellular glutamate level, as well as being a source of neuroprotectants (34). Therefore, astrocytes have become the focus of ischemia research (35). Although certain studies have reported that astrocytes were involved in the protective role of BYHWD in cerebral ischemia (13), to the best of our knowledge, no previous studies have investigated whether BYHWD directly influences astrocytes and potential pathways. The present in vitro study illustrated that astrocytic GLT-1/GS were closely associated with the protective action of BYHWD after cerebral ischemia, suggesting that astrocytes may also be the target of BYHWD besides neurons. Furthermore, it was observed that BYHWD inhibited astrocytic activation indicated by GFAP expression and the opposite association existing between GLT-1/GS and GFAP. The current study results were similar with the findings of previous studies, which indicated that upregulation of GLT-1 by ceftriaxone blocked the elevated GFAP expression, while downregulation occurred in activated astrocytes with high levels of GFAP $(36,37)$. 
In conclusion, the present study was the first to demonstrate that BYHWD served a protective role against OGD/R through upregulation of the p38 MAPK-activated GLT-1/GS signaling pathway in cultured astrocytes. These findings not only provided further evidence that astrocytes are involved in the protection of BYHWD after ischemic injury, but also revealed that BYHWD can directly affect astrocytes rather than through neurons, which may provide novel insight into the mechanisms of BYHWD.

\section{Acknowledgements}

The present study was supported by grants from the National Nature Science Foundation of China (nos. 81202817 and 81100827), the Special Research Fund for the Doctoral Program of Higher Education (no. 20124425120011), the Guangdong Province Outstanding Young Teachers in Higher Education Institutions (no. Yq2013041), and the Guangdong Natural Science Foundation (nos. S2012040007519 and S2011040002763).

\section{References}

1. Sun J, Bi Y, Guo L, Qi X, Zhang J, Li G, Tian G, Ren F and Li Z: Buyang huanwu decoction promotes growth and differentiation of neural progenitor cells: Using a serum pharmacological method. J Ethnopharmacol 113: 199-203, 2007.

2. Wei RL, Teng HJ, Yin B, Xu Y, Du Y, He FP, Chu KT, Luo BY and Zheng GQ: A systematic review and meta-analysis of buyang huanwu decoction in animal model of focal cerebral ischemia. Evid Based Complement Alternat Med 2013: 138484, 2013.

3. Hao CZ, Wu F, Shen J, Lu L, Fu DL, Liao WJ and Zheng GQ: Clinical efficacy and safety of buyang huanwu decoction for acute ischemic stroke: A systematic review and meta-analysis of 19 randomized controlled trials. Evid Based Complement Alternat Med 2012: 630124, 2012.

4. Yang S, Gao Q, Xing S, Feng X, Peng L, Dong H, Bao L, Zhang J, $\mathrm{Hu}$ Y, Li G, et al: Neuroprotective effects of buyang huanwu decoction against hydrogen peroxide induced oxidative injury in schwann cells. J Ethnopharmacol 137: 1095-1101, 2011.

5. Li XM, Bai XC, Qin LN, Huang H, Xiao ZJ and Gao TM: Neuroprotective effects of buyang huanwu decoction BYHWD on neuronal injury in hippocampus after transient forebrain ischaemia in rats. Neurosci Lett 346: 29-32, 2003.

6. Sun JH, Gao YM, Yang L, Wang X, Bao LH, Liu WJ and Yew D: Effects of buyang huanwu decoction on neurite outgrowth and differentiation of neuroepithelial stem cells. Chin J Physiol 50: 151-156, 2007.

7. Wang L, Huang Y, Wu J, Lv G, Zhou L and Jia J: Effect of buyang huanwu decoction on amino acid content in cerebrospinal fluid of rats during ischemic/reperfusion injury. J Pharm Biomed Anal 86: 143-150, 2013.

8. Zhao LD, Wang JH, Jin GR, Zhao Y and Zhang HJ: Neuroprotective effect of buyang huanwu decoction against focal cerebral ischemia/reperfusion injury in rats-time window and mechanism. J Ethnopharmacol 140: 339-344, 2012.

9. Kostandy BB: The role of glutamate in neuronal ischemic injury: The role of spark in fire. Neurol Sci 33: 223-237, 2012.

10. Verma R, Mishra V, Sasmal D and Raghubir R: Pharmacological evaluation of glutamate transporter 1 (GLT-1) mediated neuroprotection following cerebral ischemia/reperfusion injury. Eur J Pharmacol 638: 65-71, 2010.

11. Harvey BK, Airavaara M, Hinzman J, Wires EM, Chiocco MJ, Howard DB, Shen H, Gerhardt G, Hoffer BJ and Wang Y: Targeted over-expression of glutamate transporter 1 (GLT-1) reduces ischemic brain injury in a rat model of stroke. PLoS One 6: e22135, 2011.

12. Lee A, Lingwood BE, Bjorkman ST, Miller SM, Poronnik P, Barnett NL, Colditz P and Pow DV: Rapid loss of glutamine synthetase from astrocytes in response to hypoxia: Implications for excitotoxicity. J Chem Neuroanat 39: 211-220, 2010.
13. Zou J, Wang YX, Dou FF, Lü HZ, Ma ZW, Lu PH and Xu XM: Glutamine synthetase down-regulation reduces astrocyte protection against glutamate excitotoxicity to neurons. Neurochem Int 56: 577-584, 2010.

14. Ding W, Yu P, Liu W, Zhou L, Guan L and Lin R: Buyang huanwu decoction increases the expression of glutamate transporter-1 and glutamate synthesase in association with PACAP-38 following focal ischemia. Biomed Rep 3: 651-656, 2015.

15. Anlauf $E$ and Derouiche A: Glutamine synthetase as an astrocytic marker: Its cell type and vesicle localization. Front Endocrinol (Lausanne) 4: 144, 2013.

16. Shaw LH, Lin LC and Tsai TH: HPLC-MS/MS analysis of a traditional Chinese medical formulation of Bu-Yang-Huan-Wu-Tang and its pharmacokinetics after oral administration to rats. PLoS One 7: e43848, 2012.

17. Irving EA and Bamford $\mathrm{M}$ : Role of mitogen- and stress-activated kinases in ischemic injury. J Cereb Blood Flow Metab 22: 631-647, 2002.

18. Jayakumar AR, Panickar KS, Murthy ChR and Norenberg MD: Oxidative stress and mitogen-activated protein kinase phosphorylation mediate ammonia-induced cell swelling and glutamate uptake inhibition in cultured astrocytes. J Neurosci 26: 4774-4784, 2006.

19. Matos M, Augusto E, Oliveira CR and Agostinho P: Amyloid-beta peptide decreases glutamate uptake in cultured astrocytes: Involvement of oxidative stress and mitogen-activated protein kinase cascades. Neuroscience 156: 898-910, 2008.

20. Chen A, Wang H, Zhang J, Wu X, Liao J, Li H, Cai W, Luo $\mathrm{X}$ and Ju G: BYHWD rescues axotomized neurons and promotes functional recovery after spinal cord injury in rats. J Ethnopharmacol 117: 451-456, 2008.

21. Liu W, Yang ZL, Zhou LQ, Li XY, Yan FM, Guan L, Liu HM and Feng JQ: Morphine treatment enhances extracellular ATP enzymolysis and adenosine generation in rat astrocytes. Sheng Li Xue Bao 63: 20-24, 2011.

22. Begum G, Kintner D, Liu Y, Cramer SW and Sun D: DHA inhibits ER Ca2 ${ }^{+}$release and ER stress in astrocytes following in vitro ischemia. J Neurochem 120: 622-630, 2012.

23. Pérez-de la Mora M, Méndez-Franco J, Salceda R and Riesgo-Escovar JR: A glutamate dehdrogenase-based method for the assay of L-glutamic acid: Formation of pyridine nucleotide fluorescent derivitives. Anal Biochem 180: 248-252, 1989.

24. Rothstein JD, Patel S, Regan MR, Haenggeli C, Huang YH, Bergles DE, Jin L, Dykes Hoberg M, Vidensky S, Chung DS, et al: Beta-lactam antibiotics offer neuroprotection by increasing glutamate transporter expression. Nature 433: 73-77, 2005.

25. Gong SJ, Chen LY, Zhang M, Gong JX, Ma YX, Zhang JM, Wang YJ, Hu YY, Sun XC, Li WB and Zhang Y: Intermittent hypobaric hypoxia preconditioning induced brain ischemic tolerance by up-regulating glial glutamate transporter-1 in rats. Neurochem Res 37: 527-537, 2012.

26. Liu AJ, Hu YY, Li WB, Xu J and Zhang M: Cerebral ischemic pre-conditioning enhances the binding characteristics and glutamate uptake of glial glutamate transporter-1 in hippocampal CA1 subfield of rats. J Neurochem 119: 202-209, 2011.

27. Zhang XM, Zhang L, Wang G, Niu W, He Z, Ding L and Jia J: Suppression of mitochondrial fission in experimental cerebral ischemia: The potential neuroprotective target of p38 MAPK inhibition. Neurochem Int 90: 1-8, 2015.

28. Sugino T, Nozaki K, Takagi Y, Hattori I, Hashimoto N, Moriguchi $T$ and Nishida E: Activation of mitogen-activated protein kinases after transient forebrain ischemia in gerbil hippocampus. J Neurosci 20: 4506-4514, 2000.

29. Lennmyr F, Ericsson A, Gerwins P, Ahlström H and Terént A: Increased brain injury and vascular leakage after pretreatment with p38-inhibitor SB203580 in transient ischemia. Acta Neurol Scand 108: 339-345, 2003.

30. Zheng S and Zuo Z: Isoflurane preconditioning induces neuroprotection against ischemia via activation of P38 mitogen-activated protein kinases. Mol Pharmacol 65: 1172-1180, 2004.

31. Ye Z, Guo Q, Wang N, Xia P, Yuan Y and Wang E: Delayed neuroprotection induced by sevoflurane via opening mitochondrial ATP-sensitive potassium channels and p38 MAPK phosphorylation. Neurol Sci 33: 239-249, 2012.

32. Guan J, Li H, Lv T, Chen D, Yuan Y and Qu S: Bone morphogenic protein-7 contributes to cerebral ischemic preconditioning induced-ischemic tolerance by activating p38 mitogen-activated protein kinase signaling pathway. Inflammation 37: 1289-1296, 2014. 
33. Zhao L, Liu X, Liang J, Han S, Wang Y, Yin Y, Luo Y and Li J: Phosphorylation of p38 MAPK mediates hypoxic preconditioning-induced neuroprotection against cerebral ischemic injury via mitochondria translocation of $\mathrm{Bcl}-\mathrm{xL}$ in mice. Brain Res 1503: 78-88, 2013.

34. Gabryel B, Kasprowska D, Kost A, Łabuzek K and Urbanek T: Astrocytes in ischemic stroke - a potential target for neuroprotective strategies. Postepy Hig Med Dosw (Online) 69: 384-397, 2015 (In Polish).

35. Choudhury GR and Ding S: Reactive astrocytes and therapeutic potential in focal ischemic stroke. Neurobiol Dis 85: 234-244, 2016.
36. Ramos KM, Lewis MT, Morgan KN, Crysdale NY, Kroll JL, Taylor FR, Harrison JA, Sloane EM, Maier SF and Watkins LR: Spinal upregulation of glutamate transporter GLT-1 by ceftriaxone: Therapeutic efficacy in a range of experimental nervous system disorders. Neuroscience 169: 1888-1900, 2010.

37. Goodrich GS, Kabakov AY, Hameed MQ, Dhamne SC, Rosenberg PA and Rotenberg A: Ceftriaxone treatment after traumatic brain injury restores expression of the glutamate transporter, GLT-1, reduces regional gliosis, and reduces post-traumatic seizures in the rat. J Neurotrauma 30: 1434-1441, 2013. 